\title{
A Jornada Mundial da Juventude 2013: os impactos econômicos dos gastos dos peregrinos na Cidade do Rio de Janeiro
}

\section{The World Youth Day 2013: The economic impacts of spending pilgrims in Rio de Janeiro City}

\section{João Evangelista Dias Monteiro}

Universidade Federal Fluminense/Faculdade de Turismo e Hotelaria/Departamento de Turismo- Campus do Gragoatá - Rua Prof. Marcos Valdemar de Freitas Reis, s/n, Bloco H - 117 - Gragoatá, Niterói, Rio de Janeiro, Brasil, joaoedm@turismo.uff.br

\section{Osiris Ricardo Bezerra Marques}

Universidade Federal Fluminense/Faculdade de Turismo e Hotelaria/Departamento de Turismo- Campus do Gragoatá - Rua Prof. Marcos Valdemar de Freitas Reis, s/n, Bloco H - 117 - Gragoatá, Niterói, Rio de Janeiro, Brasil, osirismarques@id.uff.br

\section{Resumo}

0 mercado de eventos representa um dos principais impulsionadores do turismo e, por conseguinte, uma fonte importante de geração de negócios, emprego e renda para as cidades e países. Para avaliar a relevância econômica dos eventos, tem-se desenvolvido alguns modelos que permitem mensurar os seus impactos. Este artigo tem como objetivo quantificar os impactos econômicos positivos da Jornada Mundial da Juventude - JMJ, realizada na cidade do Rio de Janeiro, em 2013. Utilizando os dados coletados através de uma pesquisa com uma amostra de 1800 peregrinos, durante a realização do Evento e, a combinação de um modelo de impacto regional para quantificar os impactos diretos com um modelo insumo-produto para estimar os impactos indiretos, foi possível estimar o valor financeiro gerado pelos turistas nacionais e internacionais que vieram participar do evento. 0 impacto total foi estimado em aproximadamente $\mathrm{R} \$ 1,9$ bilhão, sendo $\mathrm{R} \$ 920$ milhões de impacto direto e R\$980 milhões de impacto indireto, o que reforça a importância dos eventos no processo de geração de renda para as cidades que as recebem.

Palavras chave: Impacto econômico do turismo, eventos, Rio de Janeiro, Jornada Mundial da Juventude.

\begin{abstract}
The events market is a key driver of tourism and therefore an important source of business, employment and income generation for cities and countries. Some models have been developed to evaluate the economic relevance of the events and to measure its impacts. This article aims to quantify the positive economic impacts of World Youth Day - WYD, held in the city of Rio de Janeiro in 2013. Using data collected through a survey from a sample of 1800 pilgrims during the event and the combination of a regional impact model to quantify the direct impact with an input-output model to estimate the indirect impacts it was possible to estimate the financial value generated by national and international tourists who came to attend the event. The total impact is estimated at approximately $\mathrm{R} \$ 1.9$ billion, $\mathrm{R} \$ 920$ million direct impact and R \$980 million indirect impact, which reinforces the importance of the events in the process of income generation for the cities that receive them.
\end{abstract}

Keywords: Economic impact of tourism, events, Rio de Janeiro, World Youth Day.

\section{Introdução}

0 mercado de eventos representa um dos principais impulsionadores do turismo e, por conseguinte, uma fonte importante de geração de negócios, emprego e renda nas cidades e países receptores.

A cidade do Rio de Janeiro, além de ocupar uma posição importante em relação ao turismo de lazer, nos últimos anos tem se destacado como uma das principais destinos nacionais na captação de megaeventos internacionais.

Em 2013, o Rio de Janeiro recebeu vários eventos internacionais que foram responsáveis por uma parte expressiva dos turistas que visitaram a cidade, além de gerarem impacto de aproximadamente $\mathrm{R} \$ 3$ bilhões na economia local (Observatório de Turismo da UFF, 2013).

Os impactos desses eventos podem e devem ser mensurados para que se tenha sua dimensão econômica no processo de geração de negócios, renda e emprego, para a cidade receptora.

A literatura sobre a análise dos impactos econômicos do turismo, incluindo o segmento de eventos, destaca não só os impactos positivos, como os impactos negativos que a realização de eventos pode gerar para as cidades receptoras. Também existe uma preocupação em relação ao uso inadequado dos modelos de avaliação de impactos e do conceito de multiplicador (Dwyer, Forsyth, \& Spurr, 2004; Frechtling, 1994; Archer, 1984; Fletcher, 1989).

A Jornada Mundial da Juventude (JMJ) é um evento religioso da Igreja Católica instituído no final de 1985 pelo Papa João Paulo II, que reúne milhões de jovens católicos de todo o mundo. 0 evento dura cerca de uma semana e promove uma série de atividades e encontros dos jovens na cidade sede, com objetivo de disseminar a doutrina católica e estreitar os laços de católicos de todos os continentes e de diferentes culturas. Para cada Jornada, o Papa sugere um tema, retirado de um versículo bíblico.

A primeira edição do evento ocorreu em Roma, na Itália, em 23 de março de 1986. Posteriormente, ainda sob o comando do Papa João Paulo II, o evento ocorreu em Buenos Aires (Argentina), em 1987; em Santiago de Compostela (Espanha), em 1989; na Czestochowa (Polônia), em 1991; em Denver (Estados Unidos), em 1993; em Manila (Filipinas), em 1995; em Paris (França), em 1997; novamente em Roma (Itália), em 2000 e em Toronto (Canadá), em 2002. Já sob a égide do Papa Bento XVI, o evento ocorreu em Colônia (Alemanha), em 2005; em Sydney (Austrália), em 2008 e em Madrid (Espanha), em 2011. Finalmente, com o Papa Francisco no comando da 
Igreja Católica, a Jornada Mundial da Juventude ocorreu no Rio de Janeiro (Brasil), em 2013.

Cada uma das edições da JMJ atrai milhares de peregrinos para a cidade anfitriã, mobilizando o destino na esfera cultural, social e econômica. Especialmente na esfera econômica, objeto de análise deste artigo, é possível verificar uma importante injeção de recursos na economia local por conta dos gastos dos peregrinos com alimentação, hospedagem, transporte, visitação de atrativos, compras, dentro outros gastos diversos. Nesse sentido, o objetivo deste artigo é quantificar os impactos econômicos positivos da Jornada Mundial da Juventude ocorrida na Cidade do Rio de Janeiro no ano de 2013. Vale destacar que foi a primeira JMJ capitaneada pelo Papa Francisco, primeiro Papa sulamericano, o que despertou grande interesse dos católicos desse continente em participar do evento.

Para tanto, utiliza-se neste artigo uma combinação de dois modelos de avaliação de impacto econômico do turismo: um modelo de impacto regional para quantificar os impactos diretos dos eventos e um modelo insumo-produto para avaliar os impactos indiretos.

\section{Analisando os impactos econômicos de eventos}

Os eventos possuem potencial para gerar tanto impactos positivos como negativos sobre a economia receptora. Dentre os impactos positivos podem ser elencados: aumento do fluxo de visitantes para a cidade receptora; geração de empregos temporários e permanentes; renda; impostos para o governo e; incremento na competitividade turística, principalmente quando se trata de megaeventos como a Copa do Mundo - FIFA e as Olimpíadas. Por outro lado, os eventos geram alguns transtornos e perdas para a comunidade local. No caso dos eventos de pequeno porte, os impactos negativos podem ser negligenciados, mas quando se trata de Megaeventos, os impactos negativos podem ser relevantes.

Entre os aspectos negativos destacam-se, o custo de oportunidade dos investimentos realizados na preparação da cidade ou país para receber o evento, o aumento de preços gerado pelo aumento na demanda de produtos e serviços, especulação imobiliária, entre outros. Hall (1992) enumerou os potenciais impactos positivos e negativos dos megaeventos sobre a comunidade receptora (gráfico 1).

\section{Gráfico 1 - Relação entre o tamanho dos eventos e os seus impactos}

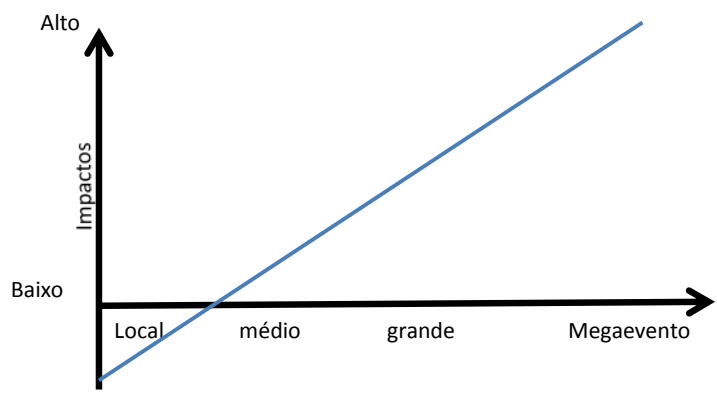

Fonte: McDonnell, I., Allen, J. \& O’Toole, W. (1999)

Pela observação do gráfico 1, percebe-se que quanto maior o evento, maiores serão os seus potenciais impactos positivos e negativos. Entre os indicadores que podem ser utilizados para avaliar o sucesso do evento estão as variáveis quantitativas e as qualitativas. Dentre as variáveis quantitativas citam-se: a estada média do turista na cidade da realização do evento, ou seja, avaliar se houve um aumento na média de dormidas por turistas; gasto médio dos visitantes e quantidade de turistas. Como variáveis qualitativas pode-se utilizar: melhoria na competitividade dos serviços, ou seja, se houve ou não melhoria na qualidade dos serviços ofertados e melhoria no sentimento cívico ou de solidariedade da comunidade receptora. (Janeczko, Ben, 2002)

Como destacado por Hall (1992), os impactos de um evento podem ser divididos em: econômicos, turismo/comercial, físico/construção, social/cultural, psicológico e político administrativo.

Neste trabalho são abordados apenas os impactos econômicos positivos associados ao turismo resumidos no quadro 1.

Quadro 1 - Os Impactos positivos e negativos dos eventos

\begin{tabular}{|c|c|c|}
\hline & Positivos & Negtivos \\
\hline \multirow{4}{*}{ Econômicos } & Crescimento das despesas de consumo & Aumento dos Preços \\
\hline & Geração de emprego & Especulação no mercado imobiliário \\
\hline & Crescimento na oferta de trabalho & Custo de oportunidade do evento \\
\hline & Melhoria no padrão de vida da comunidade receptora & $\begin{array}{l}\text { Estimativa inadequada dos custos do } \\
\text { evento }\end{array}$ \\
\hline \multirow{4}{*}{$\begin{array}{l}\text { Turismo/ } \\
\text { comércio }\end{array}$} & Crescimento do fluxo de turistas para a região & \multirow{4}{*}{$\begin{array}{c}\text { Perda de reputação associado a } \\
\text { problemas ocorridos durante a realização } \\
\text { do evento }\end{array}$} \\
\hline & $\begin{array}{l}\text { Divulgação do destino como potencial para receber } \\
\text { investimentos e desenvolvimento de atividades comercias }\end{array}$ & \\
\hline & Construção de novos hotéis e criação de novos atrativos & \\
\hline & Melhoria no acesso & \\
\hline
\end{tabular}

Fonte: Hall 1992: 
Como se pode constatar no quadro 1, apesar dos impactos econômicos positivos, abordados no trabalho, os eventos também geram impactos negativos sobre as economias receptoras, entre os quais destaca-se o aumento da inflação e a especulação imobiliária.

Como mencionado anteriormente, os impactos potenciais dependem do tamanho do evento. No caso da JMJ, caracterizado como um megaevento, que exige uma preparação da cidade para receber milhares de visitantes em um período de uma semana, tem um potencial de impactos econômicos bastante expressivo.

É importante ressaltar que apesar de ser classificado como um megaevento, a JMJ tem características diferentes dos megaeventos como a Copa do Mundo - FIFA e as Olimpíadas, caracterizadas por transformações estruturais importantes na dinâmica das cidades sedes.

Os impactos dos megaeventos podem ser divididos em três etapas com características diferentes. A primeira etapa corresponde ao período pré-evento, caracterizado pelos impactos dos investimentos na preparação da cidade ou país para receber o evento (Solberg \& Preuss, 2007); a segunda etapa corresponde ao período de realização do evento, marcado pelos impactos dos gastos dos participantes do evento nos diversos serviços e produtos e, finalmente, o legado do evento que corresponde aos impactos no período pós-evento.

No caso da JMJ, objeto de estudo deste artigo, tem os seus impactos econômicos concentrados no período da realização do evento, uma vez que, não exige grandes investimentos no período pré-evento, mas por outro lado, traz milhares de visitantes para a cidade sede num período de uma semana.

\section{Modelos de avaliação de impactos econômicos do turismo}

Para a mensuração dos impactos econômicos dos eventos são utilizados os mesmos modelos empregados na quantificação dos impactos econômicos do turismo. A complexidade da cadeia produtiva do turismo reflete-se diretamente nos modelos usados na mensuração dos seus impactos econômicos. Em relação aos modelos, os mais usados para a mensuração dos impactos do turismo são: os modelos input-output, caracterizado por ser um modelo que faz uso da matriz de insumo-produto das contas nacionais e os Modelos de Equilíbrio Geral Computável.

Neste trabalho é utilizada uma combinação de dois modelos de avaliação de impacto econômico do turismo: um Modelo de impacto regional para quantificar os impactos diretos dos gastos dos peregrinos da JMJ 2013 e um modelo insumo-produto para avaliar os seus impactos indiretos.

\section{Segundo Fletcher (1989, p.515):}

O impacto econômico do turismo é complexo, pois não ocorre no âmbito de um único setor industrial comumente reconhecido. Há uma variedade de métodos que podem ser utilizados no estudo de impacto do turismo e a escolha definitiva da metodologia é, em grande medida, determinada pelo objetivo principal da pesquisa, disponibilidade de recursos para o estudo, o tempo de restrição imposta aos investigadores, e a estrutura da economia em questão.
O modelo input-output (doravante I-0) é um modelo matemático que descreve os fluxos financeiros entre setores dentro da economia de uma região, país ou cidade. Os fluxos são previstos para saber o que cada setor deve comprar dos outros setores da economia para produzir um Real de produto. Usando a função de produção de cada atividade produtiva, os modelos I-O também permitem determinar as proporções de vendas que são destinados a salários, lucros e impostos. Os multiplicadores podem ser estimados a partir de modelos de insumo-produto com base na recirculação estimada de gastos na área avaliada. Exportações e importações são determinadas com base nas estimativas da propensão das famílias e das empresas pertencentes à região para adquirir bens e serviços a partir de fontes locais.

Quanto mais a autossuficiência da região em relação a compras de bens e serviços, maiores serão os multiplicadores para a região. (Stynes, 1999)

Os modelos de IO são fundamentados no sistema de contas nacionais, que se baseia em um sistema padrão de classificação industrial (códigos SIC) e vários censos econômicos do governo federal, que fornecem informações sobre as empresas, demanda das famílias, pagamentos de salários e geração de emprego.

Segundo Stynes (1999), apesar do modelo insumo-produto apresentar certa defasagem temporal em relação ao período de análise, este não é geralmente um grande problema, a menos que a economia da região tenha mudado significativamente. Um modelo IO representa a economia da região em um determinado ponto no tempo.

Esse modelo permite identificar as relações entre os setores da economia e o turismo e, por conseguinte, a mensuração dos impactos dos gastos dos turistas sobre a economia de uma cidade, região ou país. Através das relações Intersetoriais é possível calcular os multiplicadores que permitem quantificar os impactos indiretos dos gastos dos turistas nas atividades características.

Para avaliar os impactos indiretos é necessário utilizar os multiplicadores de cada uma das atividades que oferecem serviços e produtos diretamente para os turistas. Os multiplicadores dependem das relações entre cada uma das atividades características de turismo e a sua respectiva cadeia produtiva. Por Exemplo, o multiplicador de renda da atividade alojamento, mede o impacto que cada unidade monetária gastos pelo turista em hospedagem gera de renda, na cadeia produtiva de alojamento.

É importante ressaltar que, apesar da opção pelo uso do modelo de Insumo-produto, reconhece-se a importância e a capacidade de outros modelos utilizados para estimar os impactos do turismo. Os Modelos de Equilíbrio Geral Computável (EGC) também tem sido muito utilizado para estimar os impactos do turismo, principalmente na Austrália e no Reino Unido. (Dwyer et al., 2004).

\subsection{Multiplicadores do turismo}

Os multiplicadores foram construídos a partir do modelo insumo-produto, utilizando a matriz Leontief, das contas nacionais. 0 modelo insumo-produto foi desenvolvido pelo 
economista Wassily Leontief e apresentado originalmente, em 1941, no livro, "The Structure of the American Economy". 0 modelo é um instrumento da contabilidade social que permite analisar como as alterações na demanda de um determinado setor podem afetar os outros setores da economia. Sendo assim, a matriz insumo-produto de Leontief pode ser representada da seguinte forma:

$$
B=(I-A)^{-1},
$$

onde I é a matriz identidade e A é matriz dos coeficientes técnicos diretos.

A matriz A fornece o impacto direto derivado do aumento na demanda final, enquanto que (I - A) ${ }^{-1}$, além dos efeitos diretos, avalia também os indiretos. Desta forma, o modelo Leontief permitirá avaliar o impacto total que uma variação na demanda final de uma atividade causará na produção da economia como um todo.

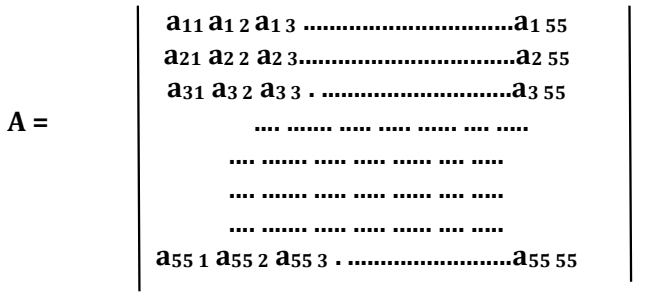

Cada coeficiente técnico é calculado a partir da fórmula: $\mathrm{a}_{\mathrm{ij}}=$ $\mathrm{c}_{\mathrm{ij}} / \mathrm{g}_{\mathrm{j}}$, onde: $\mathrm{a}_{\mathrm{ij}}$ é o coeficiente técnico do setor $\mathrm{j}$, cij o consumo intermediário do produto i para o setor j e $\mathrm{g}_{\mathrm{j}} \mathrm{o}$ valor bruto da produção do setor j.

Para calcular os multiplicadores do turismo foi utilizando a matriz Leontief de 2005, do Instituto Brasileiro de Geografia e Estatística (IBGE), e os dados das contas nacionais.

É importante destacar que nas Contas Nacionais, as atividades características do turismo aparecem na Classificação Nacional das Atividades Econômicas - CNAE, agrupadas com outras atividades, ou seja, não possuem CNAE próprios e individualizados, o que exigiu alguns ajustes nas informações disponíveis para que fosse possível a construção dos multiplicadores do turismo.

\section{Os impactos econômicos da jornada mundial da juventude 2013 na cidade do Rio de Janeiro}

A Jornada Mundial da Juventude, realizada na cidade do Rio de Janeiro, entre os dias, 15 e 30 de julho e 2013, teve a participação de aproximadamente 1,5 milhão de peregrinos, entre residentes e não residentes da cidade, gerando impactos econômicos importantes para a economia do município. Pesquisa realizada pelo Observatório do Turismo do Rio de Janeiro, da Universidade Federal Fluminense, durante os dias do evento, levantou informações sobre o perfil e os gastos dos peregrinos não residentes, que visitaram a cidade, motivados pelo evento. As informações coletadas possibilitaram a mensuração dos impactos diretos e indiretos sobre a economia da cidade.

A amostra consistiu de 1800 questionários, aplicados durantes os dias do evento. No questionário, além das informações referentes à quantidade de peregrinos, estada média e gasto médio, utilizadas na quantificação dos impactos econômicos diretos e indiretos do evento, objeto deste artigo, foram levantadas informações sobre o perfil dos peregrinos, como procedência, estado civil, renda média, entre outras.

4.1 Os impactos econômicos diretos da jornada mundial da juventude 2013 na cidade do Rio de Janeiro

Os impactos diretos são caracterizados pelos gastos realizados pelos turistas nos estabelecimentos que fornecem bens e serviços turísticos, ou seja, nas atividades como: alojamento, alimentação, transporte, comércio, entre outros.

Os impactos econômicos diretos podem ser mensurados através da equação 1 seguinte: Impactos Diretos = Número de Peregrinos x Número de
Dormidas x Gasto Médio Diário

Em relação ao número de peregrinos, foram considerados 1.500.000 participantes do evento, dado fornecido pela organização do evento e, com os dados da pesquisa que apontaram para $88 \%$ de não residentes, chega-se a um total de 1.320 .000 visitantes.

Por sua vez, os visitantes foram divididos em peregrinos nacionais e internacionais. Segundo os dados da pesquisa dos 1.320 .000 visitantes, $62,00 \%$ eram peregrinos nacionais e $38,00 \%$ internacionais, o que corresponde a, 818.400 e 501.600 , respectivamente. No que se refere ao número de dormidas, os peregrinos nacionais permaneceram em média 9 dias na cidade e os estrangeiros, 11,5 dias.

A maioria dos estrangeiros peregrinos nacionais era proveniente do Estado de São Paulo e os internacionais dos Estados Unidos, como mostram os gráficos 2 e 3.

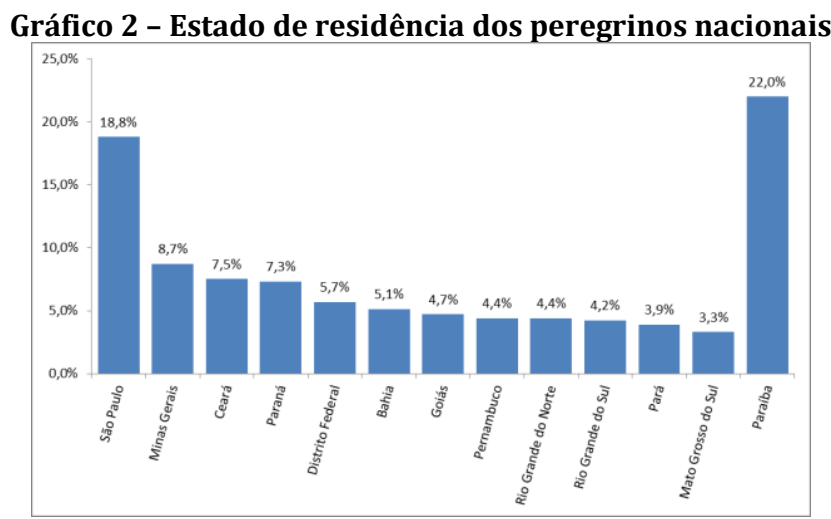

Fonte: Observatório do Turismo da UFF (2013). 


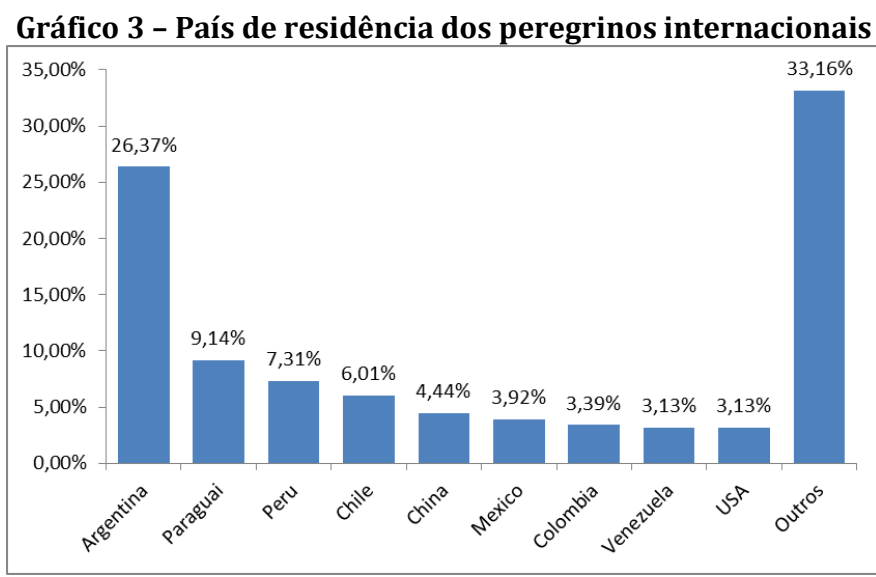

Fonte: Observatório do Turismo da UFF (2013).

Em relação aos gastos, o gasto médio diário dos peregrinos nacionais e internacionais foi de, $\mathrm{R} \$ 48,71$ e $\mathrm{R} \$ 81,27$, respectivamente. A consolidação destas informações está representada na tabela 1.

Tabela 1 - Número de peregrinos, dormidas e gasto médio diário

\begin{tabular}{|l|c|c|}
\hline \multicolumn{1}{|c|}{ Peregrinos } & Nacionais & Internacionais \\
\hline Número de Peregrinos & 818.000 & 501.600 \\
\hline Média de Dormidas (dias) & 9,0 & 11,5 \\
\hline Gasto Médio Diário & $\mathrm{R} \$ 56,80$ & $\mathrm{R} \$ 86,82$ \\
\hline
\end{tabular}

Utilizando os dados da tabela 1, e a equação 1, é possível calcular o impacto direto dos gastos dos peregrinos sobre a economia da cidade do Rio de Janeiro, como apresentado na tabela 2.

Tabela 2 - Total de impacto direto dos gastos dos peregrinos

\begin{tabular}{|c|c|c|c|}
\hline & Peregrinos Nacionais & Peregrinos Internacionais & Total Geral do Evento \\
\hline Impacto Direto & $\mathrm{R} \$ 418.340 .058,80$ & $\mathrm{R} \$ 500.802 .233,42$ & $\mathrm{R} \$ 919.142 .292,21$ \\
\hline
\end{tabular}

Utilizando a estrutura de gastos do turista de eventos do Rio de Janeiro (Observatório de Turismo da UFF, 2013) foi possível distribuir os impactos diretos entre os serviços e produtos turísticos, como mostra a tabela 3 .

Tabela 3 - Total de impacto direto dos gastos dos peregrinos

\begin{tabular}{|l|c|c|c|}
\hline \multicolumn{1}{|c|}{ Gastos por categoria } & Peregrinos Nacionais & Peregrinos Internacionais & Total \\
\hline Gasto com hospedagem & $\mathrm{R} \$ 160.203 .072,09$ & $\mathrm{R} \$ 201.770 .481,14$ & $\mathrm{R} \$ 361.973 .553,23$ \\
\hline Gasto com Alimentos & $\mathrm{R} \$ 152.308 .048,98$ & $\mathrm{R} \$ 116.477 .183,08$ & $\mathrm{R} \$ 268.785 .232,06$ \\
\hline Gasto com compras & $\mathrm{R} \$ 58.200 .850,87$ & $\mathrm{R} \$ 75.871 .352,12$ & $\mathrm{R} \$ 134.072 .202,99$ \\
\hline Gasto em atrações & $\mathrm{R} \$ 47.628 .086,85$ & $\mathrm{R} \$ 106.683 .217,08$ & $\mathrm{R} \$ 154.311 .303,93$ \\
\hline \multicolumn{1}{|c|}{ Total } & $\mathbf{R} \mathbf{4 1 8 . 3 4 0 . 0 5 8 , 8 0}$ & $\mathbf{R} \mathbf{5 0 0 . 8 0 2 . 2 3 3 , 4 2}$ & $\mathbf{R} \mathbf{9 1 9 . 1 4 2 . 2 9 2 , 2 1}$ \\
\hline
\end{tabular}

Fonte: Elaboração dos autores.

Os impactos diretos totalizaram $\mathrm{R} \$ 919.142 .292,21$, sendo $\mathrm{R} \$ 418.340 .058,80$ gerados pelos peregrinos nacionais e $\mathrm{R} \$$ $500.802 .233,42$, pelos peregrinos internacionais. Apesar do maior gasto com o serviço de hospedagem, é importante ressaltar que a maioria dos visitantes ficou hospedado em casa de amigos e parentes, escolas, igrejas, entre outros meios de hospedagem alternativos.

\subsection{Os impactos econômicos diretos e indiretos da jornada mundial da juventude 2013 na cidade do Rio de Janeiro}

Devido à inexistência de informações de uma matriz insumo-produto estadual ou municipal, para o cálculo do impacto total (Impactos diretos e indiretos) dos gastos dos peregrinos, foram utilizados os multiplicadores calculados a partir do modelo Insumo-Produto, com os dados das contas nacionais do IBGE.

Os multiplicadores apresentados na tabela 4, representam o quanto é gerado na cadeia produtiva de cada atividade para cada $\mathrm{R} \$ 1,00$ de gasto direto dos peregrinos na compra dos produtos e serviços turísticos. Por exemplo, cada R\$1,00 gasto no serviço de hospedagem, gera $\mathrm{R} \$ 2,13$ na cadeia produtiva da atividade de alojamento. 
Tabela 4 - Os multiplicadores das atividades turísticas

\begin{tabular}{|l|c|}
\hline \multicolumn{1}{|c|}{ Gastos por categoria } & Multiplicador \\
\hline Gasto com hospedagem & 2,13 \\
\hline Gasto com Alimentos & 2,13 \\
\hline Gasto com compras & 2,01 \\
\hline Gasto em atrações & 1,86 \\
\hline
\end{tabular}

Fonte: Elaboração dos autores/Contas Nacionais do IBGE.

Assim, para avaliar os impactos totais, ou seja, diretos e indiretos foi utilizada a equação 2 seguinte.

Impacto Total = Multiplicador * Impacto Direto
Utilizando os multiplicadores apresentados na tabela 4 e os dados de impacto direto da tabela 3 , determina-se os impactos econômicos totais (direto e indireto) dos gastos dos peregrinos sobre as atividades características de turismo na cidade do Rio de Janeiro.

A tabela 5 contém os impactos econômicos diretos e indiretos dos gastos dos peregrinos sobre a economia da cidade do Rio de Janeiro, durante o evento Jornada Mundial da Juventude, realizado entre os dias 15 e 30 de julho de 2013. Os impactos diretos totalizaram $\mathrm{R} \$ 919.142 .292,21$, enquanto que, os impactos indiretos foram de $\mathrm{R} \$$ $980.878 .880 .073,78$

Tabela 5 - Os impactos totais dos gastos dos peregrinos nas atividades turísticas

\begin{tabular}{|l|c|c|c|}
\hline \multicolumn{1}{|c|}{ Gastos por categoria } & Peregrinos Nacionais & Peregrinos Internacionais & Total \\
\hline Gasto com hospedagem & $\mathrm{R} \$ 341.232 .543,56$ & $\mathrm{R} \$ 429.771 .124,83$ & $\mathrm{R} \$ 771.003 .668,38$ \\
\hline Gasto com Alimentos & $\mathrm{R} \$ 324.416 .144,33$ & $\mathrm{R} \$ 248.096 .399,95$ & $\mathrm{R} \$ 572.512 .544,28$ \\
\hline Gasto com compras & $\mathrm{R} \$ 116.983 .710,25$ & $\mathrm{R} \$ 152.501 .417,76$ & $\mathrm{R} \$ 269.485 .128,01$ \\
\hline Gasto em atrações & $\mathrm{R} \$ \mathbf{8 8 . 5 8 8 . 2 4 1 , 5 5}$ & $\mathrm{R} \$ 198.430 .783,77$ & $\mathrm{R} \$ 287.019 .025,32$ \\
\hline Total & $\mathbf{R} \mathbf{8 7 1 . 2 2 0 . 6 3 9 , 6 9}$ & $\mathbf{R} \mathbf{1 . 0 2 8 . 7 9 9 . 7 2 6 , 3 1}$ & $\mathbf{R} \mathbf{1 . 9 0 0 . 0 2 0 . 3 6 6 , 0 0}$ \\
\hline
\end{tabular}

Fonte: Elaboração dos autores.

Esses valores expressivos mostram a relevância econômica dos grandes eventos sobre a economia das cidades que os recebe. De qualquer forma, é importante ressaltar que além do impacto de gastos, tema desse artigo, os eventos, principalmente os megaeventos, geram outros impactos sobre as cidades, regiões ou países que os recebem.

\section{Conclusão}

Os eventos podem gerar impactos econômicos relevantes sobre as cidades e as comunidades que os recebem. Os impactos podem ser positivos ou até mesmo, negativos e sua intensidade depende da dimensão e da abrangência do evento.

Os impactos dos megaeventos devem ser avaliados desde a sua preparação, passando pelo período da realização até o pós-evento, possibilitando a avaliação dos legados. No caso do evento "Jornada Mundial da Juventude 2013", apesar da dimensão mundial em termos de participantes, não houve a necessidade da realização de investimentos em infraestrutura da cidade para recebê-lo. Neste sentido, os impactos econômicos do evento foram concentrados no período da sua realização, permitindo a sua mensuração a partir de levantamentos de informações primárias realizados durante o evento.

Aplicando uma pesquisa, com uma amostra estatisticamente significativa, foi possível coletar informações sobre a quantidade de peregrinos nacionais e internacionais, média de estada dos peregrinos na cidade e o gasto médio diário. Com essas informações foi possível estimar os impactos diretos dos gastos dos peregrinos que totalizaram $\mathrm{R} \$ 919.142 .292,21$. Utilizando o modelo Insumo-produto e os dados da matriz insumo-produto do IBGE, foi possível estimar os multiplicadores das atividades características de turismo. Por sua vez, com os multiplicadores e os gastos diretos, foi possível computar os impactos indiretos que totalizaram $\mathrm{R} \$ 980.878 .880 .073,78$.

\section{Referências}

Archer, B.H. (1982). The value of multiplier and their policy implications. Tourism Management, 3(4), 236-241.

Archer, B.H. (1984). Economic impact: misleading multiplier. Annals of Tourism Research, 11(3), 517-518.

Candela, Guido, Figini, Paolo (2012). The Economics of Tourism Destinations. Berlim: Springer.

Dwyer, L., Forsyth, P. \& Spurr, R. (2004). Evaluating tourism's economic effects: new and old approaches. Tourism Management, $25,307-317$.

Fletcher, J. (1989). Imput-output analysis and tourism impact studies. Annals of Tourism Research, 16(4), 514-529.

Frechtling, Douglas C. (1994). Assessing the economic impacts of travel and tourism - Measuring economic benefits. In J.R. Brent Ritchie \& Charles R. Goeldner (eds.). Travel, Tourism and Hospitality Research, second edition (367-391). New York: John Wiley and Sons Inc.

Hall, C. M., (1992). Hallmark Tourist Events: Impacts, management, and planning. London: Belhaven Press.

Instituto Brasileiro de Geografia e Estatística - IBGE (2005). Matriz de Insumo-Produto: Brasil - 2005. Rio de Janeiro: IBGE.

Janeczko, Ben (2002). Estimating the economic impacts of festivals and events: a research guide. Research report. Consultado em $15 \mathrm{de}$ maio de $2014 \mathrm{em}$ http://www.crctourism.com.au.

Kitterlin M. \& Yoo M. (2014). Festival motivation and loyalty factors. Tourism \& Management Studies, 10(1), 119-126.

Kraybill, D.S. (1993). Computable general equilibrium analysis at the regional level, in microcomputer-based input-output modeling: applications to economic development. Boulder: Westview Press.

Kronenberg, T. 2009. Construction of regional input-output models using nonsurvey methods: the role of cross-hauling. International Regional Science Review 32(1), 40-64.

Leontief, W. (1941).The structure of the American economy 19191939. New York: Oxford University Press.

McDonnell, I., Allen, J. \& O’Toole, W. (1999). Festival and special event management. Milton: Jacaranda-Wiley.

Miller, R.E., \& Blair, P.D. (1985). Input-output analysis: foundations and extensions. Englewood Cliff, NY: Prentice-Hall.

Marujo, N. (2014). Turismo e eventos: a Festa da Flor na ilha da Madeira. Tourism \& Management Studies, 10(2), 26-31.

Observatório do Turismo do Rio de Janeiro (2013). Os impactos econômicos da copa das confederações FIFA 2013 no Rio de Janeiro. Rio de Janeiro: Universidade Federal Fluminense. 
Consultado a 14 de Maio de 2014 em: http://www.observatoriodoturismo.uff.br//.

Perlich, P. (2005). Regional economic analysis and impact models. Salt Lake City, UT: Bureau of Economic and Business Research.

Solberg, H. A., \& H. Preuss. 2007. Major sport events and long-term tourism impacts. Journal of Sport Management, 21, 213-234.

Stynes, D.J. (1999). Economic impacts of tourism. Consultado a 10 de Maio de 2014 em: https://www.msu.edu/course/prr/840/econimpact/pdf/ecimpvol 1.pdf.

Submetido: 12.06 .2014

Aceite: 22.11.2014 